$\mathrm{BA}-\mathrm{TH} / 01-415$

CERN-TH/2001-149

hep-th/0106019

\title{
Localization of Scalar Fluctuations in a Dilatonic Brane-World Scenario
}

\author{
V. Bozza $\rrbracket^{(a, b)}$, M. Gasperinif( ${ }^{(c, d)}$ and G. Veneziano $]^{[(e, f)}$ \\ (a) Dipartimento di Fisica "E. R. Caianiello", Università di Salerno, \\ Via S. Allende, 84081 Baronissi (SA), Italy \\ (b) INFN, Sezione di Napoli, Gruppo Collegato di Salerno, Salerno, Italy \\ (c) Dipartimento di Fisica, Università di Bari, \\ Via G. Amendola 173, 70126 Bari, Italy \\ (d) INFN, Sezione di Bari, Bari, Italy \\ (e) Theoretical Physics Division, CERN, CH-1211 Geneva 23, Switzerland \\ (f) Laboratoire de Physique Théorique, Université Paris Sud, 91405 Orsay, France
}

\begin{abstract}
We derive and solve the full set of scalar perturbation equations for a class of $Z_{2^{-}}$ symmetric five-dimensional geometries generated by a bulk cosmological constant and by a 3-brane non-minimally coupled to a bulk dilaton field. The massless scalar modes, like their tensor analogues, are localized on the brane, and provide long-range fourdimensional dilatonic interactions, which are generically present even when matter on the brane carries no dilatonic charge. The shorter-range corrections induced by the continuum of massive scalar modes are always present: they persist even in the case of a trivial dilaton background (the standard Randall-Sundrum configuration) and vanishing dilatonic charges.
\end{abstract}

\footnotetext{
${ }^{1}$ valboz@sa.infn.it

2 gasperini@ba.infn.it

${ }^{3}$ venezia@nxth04.cern.ch
} 


\section{Introduction}

The possibility, first discovered in the context of Horava-Witten (HW) heterotic M-theory [1, 2], that our Universe could lie on a hypersurface (a "brane") embedded in some higherdimensional "bulk" space-time -the so-called brane-world scenario- has recently attracted considerable attention. In the original HW paper, our world is a 9-brane sitting at one of the boundaries of an eleven-dimensional bulk, while in a large class of M-theory models [3, 田, in which six dimensions are compactified in a more traditional Kaluza-Klein (KK) way, one can envisage constructing fully consistent four-dimensional brane-world scenarios with, effectively, a five-dimensional bulk. Unfortunately, finding brane configurations which are consistent with all stringy constraints has proved to be a very hard, if not impossible, task.

At a more phenomenological level, i.e. when problems with quantization of the higherdimensional gravity theory are ignored, one can consider the dimensions orthogonal to the 3-brane as either compact and large [5, 6, 7], or as having infinite proper size [8, 9, 10]. In the latter (so-called Randall-Sundrum (RS)) case, the bulk geometry is bent by an appropriate "warp-factor", providing a crucial difference with respect to the "old" KK scenario, where the bulk geometry is simply the direct product of an "internal" and an "external" manifold. In particular, unlike the KK models, RS models are able to reproduce the four-dimensional Newton law at large distances on the brane by dynamically binding the massless gravitons to it [10].

As a consequence of the non-factorized structure of the metric in RS-type scenarios, previous approaches to the study of metric fluctuations in higher-dimensional backgrounds [11-16], based on the isometries of a factorizable geometry, cannot be applied directly to the brane-world scenario. A new gauge-invariant formalism is required, like the one developed in [17]. The classical and quantum analysis of metric fluctuations is of primary importance for understanding the possible localization of massless modes on the brane, as well as the nature of the orrections to the long-range interaction due to the continuum of massive modes that are typically living in the bulk. Until now, the study of this problem has been mainly focused on the structure of tensor (i.e. transverse-traceless) perturbations of the bulk geometry (see [18] for a general discussion).

In all string/M-theory models, however, the graviton enjoys the company of (perturbatively) massless scalar partners (the dilaton, compactification moduli, etc.). These typically induce long-range interactions of gravitational strength [19], and are therefore dangerous in view of the existing experimental tests (see for instance [20]). The standard way to solve this problem is to assume that these scalars get a SUSY-breaking non-perturbative mass 
(for alternatives see e.g. [21]). However, one may ask whether RS-type scenarios can offer an alternative solution to (or an alleviation of) this problem, e.g. by not confining scalar fluctuations on the brane. If this were the case, scalar interactions on the brane would be suppressed, or possibly become short-ranged, and brane-world scenarios would naturally solve one of the most serious potential problems with higher-dimensional or stringy extensions of the Standard Model and General Relativity. This particular aspect of brane-world scenarios has never been completely addressed, to the best of our knowledge, in spite of many studies recently appeared in the literature, and devoted to the perturbations of a brane-world background [22]-[30].

In this paper we present a detailed discussion of the localization of scalar metric fluctuations in a typical example of brane-world scenario. Unlike gravitons, which are decoupled from matter fluctuations, the scalar fluctuations of the bulk geometry are in general non-trivially coupled to the matter sources. We shall thus consider a non-compact, $Z_{2^{-}}$ symmetric, five-dimensional background, generated by a positive tension 3-brane and by a bulk dilaton field coupled to the brane and to the (negative) bulk energy density. We shall restrict ourselves, in particular, to the gravi-dilaton solutions discussed in [31] (hereafter called CLP backgrounds, for short), which generalize the $\mathrm{AdS}_{5} \mathrm{RS}$ scenario [10] in the presence of a bulk scalar field, and which are already known to guarantee the localization of tensor metric fluctuations [31].

By extending the analysis of the perturbations, and by using an appropriate gaugeinvariant formalism [17], we find that the same class of CLP backgrounds that localize massless tensor perturbations on the four-dimensional brane also localize massless scalar perturbations (i.e. the dilatonic interaction). However, the short-range corrections to the scalar interactions, due to the massive modes propagating in the extra-dimension, are in general different from the higher-dimensional corrections affecting the pure tensor part of the gravitational interaction. We also find that, in general, scalar metric fluctuations exhibit a non-trivial, "self-sustained" spectrum of solutions, even for a trivial dilaton background. This implies that long- and short-range gravitational interactions on the brane are effectively of the scalar-tensor type, in agreement with previous results [32], and are therefore subject to strong phenomenological constraints.

The paper is organized as follows. In Section 2 we present the action and the classical equations of motion for a 3-brane non-minimally coupled to a five-dimensional gravi-dilaton background, and we retrieve the whole class of CLP solutions [31]. In Section 3 we give the full set of scalar perturbation equations in the so-called "generalized longitudinal gauge" [17], and we find the four independent canonical variables diagonalizing them. In Section 4 we discuss the localization of massless modes, and determine the class of backgrounds 
admitting long-range dilatonic interactions confined on the brane. In Section 5 we present the general spectrum of solutions for the massive modes that propagate throughout the bulk, and determine the relative magnitude of their amplitudes. In Section 6 we evaluate, in the weak field limit, the leading-order corrections to the effective gravitational potential generated by a static source with a point-like mass and dilatonic charge, confined on the brane. The main results of this paper are finally summarized in Section 7 .

\section{Background equations}

We shall consider a five-dimensional scalar-tensor background $\left\{g_{A B}, \phi\right\}$, possibly arising from the bosonic sector of a dimensionally reduced string/supergravity theory, and nontrivially coupled to a negative cosmological constant $\Lambda$ and to a 3-brane of positive tension $T_{3}$ :

$$
\begin{aligned}
S=S_{\text {bulk }}+S_{\text {brane }} & =M_{5}^{3} \int d^{5} x \sqrt{|g|}\left(-R+\frac{1}{2} g^{A B} \partial_{A} \phi \partial_{B} \phi-2 \Lambda e^{\alpha_{1} \phi}\right) \\
& -\frac{T_{3}}{2} \int d^{4} \xi \sqrt{|\gamma|}\left[\gamma^{\alpha \beta} \partial_{\alpha} X^{A} \partial_{\beta} X^{B} g_{A B} e^{\alpha_{2} \phi}-2\right] .
\end{aligned}
$$

Here $M_{5}$ is the fundamental mass scale of the five-dimensional bulk space-time, and the parameters $\alpha_{1}, \alpha_{2}$ control the coupling of the bulk dilaton to $\Lambda$ and to the brane (particular values of these parameters may simply correspond to the rescaling of the minimally coupled $\sigma$-model action in the canonical Einstein frame [33], but here we allow in general for non-minimal couplings). The brane action (see for instance [34]) is parametrized by the coordinates $X^{A}(\xi)$ describing the embedding of the brane in the bulk manifold, and by the auxiliary metric tensor $\gamma_{\alpha \beta}(\xi)$ defined on the four-dimensional world-volume of the brane, spanned by the coordinates $\xi^{\alpha}$. Consequently, $\partial_{\alpha} X^{A}$ is a short-cut notation for $\partial X^{A}(\xi) / \partial \xi^{\alpha}$.

Conventions: Greek indices run from 0 to 3, capital Latin indices from 0 to 4, lower-case Latin indices from 1 to 3 . For the bulk coordinates we use the notation $x^{A}=\left(t, x^{i}, z\right)$. The metric signature is $(+,-,-,-,-)$, and the curvature tensor is defined by $R_{M N A}{ }^{B}=$ $\partial_{M} \Gamma_{N A}^{B}+\Gamma_{M P}^{B} \Gamma_{N A}^{P}-(M \longleftrightarrow N), R_{N A}=R_{M N A}{ }^{M}$.

The variation of the action with respect to $g_{A B}, \phi, X^{A}$ and $\gamma_{\alpha \beta}$ gives, respectively, the Einstein equations (in units such that $M_{5}^{3}=1$ ):

$$
\begin{aligned}
G_{B}^{A} & =\frac{1}{2}\left(\partial^{A} \phi \partial_{B} \phi-\frac{1}{2} \delta_{B}^{A} \partial_{C} \phi \partial^{C} \phi\right)+\Lambda e^{\alpha_{1} \phi} \delta_{B}^{A}+ \\
& +\frac{T_{3}}{2} \frac{1}{\sqrt{|g|}} g_{B C} \int d^{4} \xi \sqrt{|\gamma|} \delta^{5}(x-X) \gamma^{\alpha \beta} \partial_{\alpha} X^{A} \partial_{\beta} X^{C} e^{\alpha_{2} \phi}
\end{aligned}
$$


the dilaton equation:

$$
\nabla_{M} \nabla^{M} \phi+2 \alpha_{1} \Lambda e^{\alpha_{1} \phi}+\frac{\alpha_{2} T_{3}}{2} \frac{1}{\sqrt{|g|}} \int d^{4} \xi \sqrt{|\gamma|} \delta^{5}(x-X) \gamma^{\alpha \beta} \partial_{\alpha} X^{A} \partial_{\beta} X^{B} g_{A B} e^{\alpha_{2} \phi}=0
$$

the equation governing the evolution of the brane in the bulk space-time:

$$
\partial_{\alpha}\left(\sqrt{|\gamma|} \gamma^{\alpha \beta} \partial_{\beta} X^{B} g_{A B} e^{\alpha_{2} \phi}\right)=\left.\frac{1}{2} \sqrt{|\gamma|} \gamma^{\alpha \beta} \partial_{\alpha} X^{B} \partial_{\beta} X^{C} \partial_{A}\left(g_{B C} e^{\alpha_{2} \phi}\right)\right|_{x=X(\xi)}
$$

and the induced metric on the brane:

$$
\gamma_{\alpha \beta}=\partial_{\alpha} X^{A} \partial_{\beta} X^{B} g_{A B} e^{\alpha_{2} \phi}
$$

We now specialize these equations to the case of a conformally flat bulk metric, with a warp factor $a$ and a dilaton $\phi$, both of which only depend on the fifth coordinate $z$. Also, we shall look for $Z_{2}$-symmetric solutions, describing a flat brane rigidly located at $z=0$, and we set

$$
g_{A B}=a^{2}(z) \eta_{A B}, \quad \phi=\phi(z), \quad X^{A}=\delta_{\mu}^{A} \xi^{\mu} .
$$

where $\eta_{A B}$ is the five-dimensional Minkowski metric. The induced metric thus reduces to

$$
\gamma_{\alpha \beta}=\delta_{\alpha}^{A} \delta_{\beta}^{B} g_{A B} e^{\alpha_{2} \phi}
$$

while the brane equations (2.4) are identically satisfied thanks to the $Z_{2}$ symmetry.

The dynamical equations are obtained from the dilaton equation (2.3), which becomes

$$
3 \frac{a^{\prime}}{a} \phi^{\prime}+\phi^{\prime \prime}-2 \alpha_{1} \Lambda a^{2} e^{\alpha_{1} \phi}-2 \alpha_{2} T_{3} a e^{2 \alpha_{2} \phi} \delta(z)=0,
$$

and from the $(\alpha, \beta)$ and $(4,4)$ components of the Einstein equations (2.2), which give, respectively,

$$
\begin{aligned}
& -3 \frac{a^{\prime \prime}}{a}=\frac{\phi^{\prime 2}}{4}+\Lambda a^{2} e^{\alpha_{1} \phi}+\frac{T_{3}}{2} a e^{2 \alpha_{2} \phi} \delta(z), \\
& -6 \frac{a^{\prime 2}}{a^{2}}=-\frac{\phi^{\prime 2}}{4}+\Lambda a^{2} e^{\alpha_{1} \phi}
\end{aligned}
$$

(a prime denotes differentiation with respect to $z$ ). The last three equations are not independent: the dilaton equation, for instance, can be obtained by differentiating eqs. (2.9), (2.10), as a consequence of the Bianchi identities.

No general solution is known for arbitrary values of $\alpha_{1}, \alpha_{2}, T_{3}$ and $\Lambda$. However, if we fine-tune these parameters by choosing:

$$
\alpha_{1}=4 \alpha_{2}, \quad T_{3}=8 \sqrt{\Lambda / \Delta}, \quad \alpha_{1}^{2}=\Delta+\frac{8}{3},
$$


where the last equation defines $\Delta$, we recover the four-dimensional sector of a known, oneparameter family of exact domain wall solutions [35], which can be written in an explicitly $Z_{2}$-symmetric form. For $\Delta=-2$ the solution is:

$$
a(z)=e^{-\frac{k|z|}{3}}, \quad \phi(z)=\sqrt{\frac{2}{3}} k|z|, \quad k^{2}=-2 \Lambda,
$$

otherwise $(\Delta \neq-2)$ :

$$
a(z)=(1+k|z|)^{\frac{2}{3(\Delta+2)}}, \quad e^{\phi(z)}=(1+k|z|)^{-\frac{2 \alpha_{1}}{\Delta+2}}, \quad k^{2}=\frac{(\Delta+2)^{2} \Lambda}{\Delta} .
$$

We shall choose $k>0$ so that the $z$ coordinate, transverse to the brane, may run from $-\infty$ to $+\infty$ (the proper size of the transverse dimension is finite, however, unless $\Delta=-8 / 3)$. In that case, the solution corresponds to a brane of positive tension, $T_{3}>0$, provided $\Delta \leq-2$. This range of $\Delta$ guarantees a positive tension and also avoids the presence of naked singularities [31]. On the other hand, the reality of $\alpha_{1}$ requires $\Delta \geq-8 / 3$. In the rest of this paper we shall thus assume

$$
k>0, \quad-\frac{8}{3} \leq \Delta \leq-2 .
$$

We may note that in the limit $\Delta=-8 / 3$ the dilaton decouples and becomes trivial, and the solution reduces to the well studied pure $\mathrm{AdS}_{5}$ background, originally introduced to localize gravity on a 3-brane [10].

In the following section we will obtain the canonical equations governing the evolution of scalar (metric + dilaton) fluctuations around the above CLP background solutions.

\section{Scalar perturbations}

We now perturb to first order the full set of bulk equations (2.2)-(2.5), keeping the position of the brane fixed, $\delta X^{A}=0$. It is known, indeed, that the brane location can be consistently assumed to remain unchanged, to the relevant linear order, when perturbing a background of the type we are considering [17] (see [25] for a study that includes, instead, a non-vanishing deformation of the brane). We thus set

$$
\delta g_{A B}=h_{A B}, \quad \delta g^{A B}=-h^{A B}, \quad \delta \phi=\chi, \quad \delta X^{A}=0,
$$

where the indices of the perturbed fields are raised and lowered by the unperturbed metric, and the background fluctuations $h_{A B}, \chi$ are assumed to be inhomogeneous. 
The perturbation of the background equations (2.2)-(2.5) gives, respectively, the linearized equations for the Einstein tensor:

$$
\begin{aligned}
& \delta G^{A}{ }_{B}=\frac{1}{2}\left(-h^{A C} \partial_{C} \phi \partial_{B} \phi+\partial^{A} \phi \partial_{B} \chi+\partial^{A} \chi \partial_{B} \phi\right)-\frac{1}{4} \delta_{B}^{A}\left(2 \partial^{M} \partial_{M} \chi-h^{M N} \partial_{M} \phi \partial_{N} \phi\right) \\
& +\Lambda \alpha_{1} \chi e^{\alpha_{1} \phi} \delta_{B}^{A}+\frac{T_{3}}{2} \frac{1}{\sqrt{|g|}} \int d^{4} \xi \sqrt{|\gamma|} \delta^{5}(x-X) \gamma^{\alpha \beta} \partial_{\alpha} X^{A} \partial_{\beta} X^{C} e^{\alpha_{2} \phi} \\
& \times\left[h_{B C}+g_{B C}\left(-\frac{1}{2} g^{M N} h_{M N}+\frac{1}{2} \gamma^{\mu \nu} \delta \gamma_{\mu \nu}+\alpha_{2} \chi\right)\right] \\
& +\frac{T_{3}}{2} \frac{1}{\sqrt{|g|}} g_{B C} \int d^{4} \xi \sqrt{|\gamma|} \delta^{5}(x-X) \partial_{\alpha} X^{A} \partial_{\beta} X^{C} e^{\alpha_{2} \phi} \delta \gamma^{\alpha \beta},
\end{aligned}
$$

for the dilaton:

$$
\begin{aligned}
& \nabla_{M} \nabla^{M} \chi-h^{M N} \nabla_{M} \nabla_{N} \phi-g^{M N} \delta \Gamma_{M N}^{B} \partial_{B} \phi+2 \alpha_{1}^{2} \Lambda \chi e^{\alpha_{1} \phi} \\
& +\frac{\alpha_{2} T_{3}}{2} \frac{1}{\sqrt{|g|}} \int d^{4} \xi \sqrt{|\gamma|} \delta^{5}(x-X) \gamma^{\alpha \beta} \partial_{\alpha} X^{A} \partial_{\beta} X^{B} g_{A B} e^{\alpha_{2} \phi} \\
& \times\left(-\frac{1}{2} g^{M N} h_{M N}+\frac{1}{2} \gamma^{\mu \nu} \delta \gamma_{\mu \nu}+\alpha_{2} \chi\right) \\
& +\frac{\alpha_{2} T_{3}}{2} \frac{1}{\sqrt{|g|}} \int d^{4} \xi \sqrt{|\gamma|} \delta^{5}(x-X) \partial_{\alpha} X^{A} \partial_{\beta} X^{B} e^{\alpha_{2} \phi}\left(g_{A B} \delta \gamma^{\alpha \beta}+h_{A B} \gamma^{\alpha \beta}\right)=0,
\end{aligned}
$$

for the brane:

$$
\begin{aligned}
& \partial_{\alpha}\left\{\sqrt{|\gamma|} \partial_{\beta} X^{B} e^{\alpha_{2} \phi}\left[\delta \gamma^{\alpha \beta} g_{A B}+\gamma^{\alpha \beta} h_{A B}+\gamma^{\alpha \beta} g_{A B}\left(\alpha_{2} \chi+\frac{1}{2} \gamma^{\mu \nu} \delta \gamma_{\mu \nu}\right)\right]\right\}= \\
& =\frac{1}{2} \sqrt{|\gamma|} \partial_{\alpha} X^{B} \partial_{\beta} X^{C}\left[\left.\left(\delta \gamma^{\alpha \beta}+\frac{1}{2} \gamma^{\alpha \beta} \gamma^{\mu \nu} \delta \gamma_{\mu \nu}\right) \frac{\partial}{\partial x^{A}}\right|_{x=X(\xi)}\left(g_{B C} e^{\alpha_{2} \phi}\right)\right. \\
& \left.+\left.\gamma^{\alpha \beta} \partial_{A}\left(h_{B C} e^{\alpha_{2} \phi}+\alpha_{2} \chi g_{B C} e^{\alpha_{2} \phi}\right)\right|_{x=X(\xi)}\right]
\end{aligned}
$$

and for the induced metric:

$$
\delta \gamma_{\alpha \beta}=\partial_{\alpha} X^{A} \partial_{\beta} X^{B} e^{\alpha_{2} \phi}\left(h_{A B}+\alpha_{2} \chi g_{A B}\right) .
$$

Here all geometrical quantities, such as the perturbed connection $\delta \Gamma_{A B}{ }^{C}$, the perturbed scalar curvature $\delta R=-h^{A B} R_{A B}+g^{A B} \delta R_{A B}$, and so on, are computed to first order in $h_{A B}$.

By expanding around the background (2.6), it is now easy to study the propagation of the spin-2 physical degrees of freedom on the brane, represented by the transverse and traceless perturbations $\bar{h}_{i j}$,

$$
h_{A B}=a^{2} \delta_{A}^{i} \delta_{B}^{j} \bar{h}_{i j}, \quad \bar{h}_{i}^{i}=0, \quad \nabla^{i} \bar{h}_{i j}=0 .
$$

In the linear approximation, the tensor fluctuations $\bar{h}_{i j}$ are decoupled from the scalar and matter fluctuations. We can consistently set $\chi=0$, and find that the dilaton and the 
brane equations are trivially satisfied; in addition, the right-hand side of the Einstein equations (3.2) is identically vanishing, and the linearized Ricci tensor leads to the well known covariant wave equation for gravitons:

$$
\square_{5} \bar{h}_{i j} \equiv\left(\frac{\partial^{2}}{\partial t^{2}}-\frac{\partial^{2}}{\partial x^{i 2}}-\frac{\partial^{2}}{\partial z^{2}}-\frac{3 a^{\prime}}{a} \frac{\partial}{\partial z}\right) \bar{h}_{i j}=0,
$$

where $\square_{5} \equiv \nabla_{M} \nabla^{M}$ is the five-dimensional covariant d'Alembert operator, describing free propagation in the warped bulk geometry.

In this paper (also in preparation of future cosmological applications) we are primarily interested in the scalar fluctuations of the bulk metric, which are coupled to the dilaton fluctuations. Thus, we shall keep $\delta \phi=\chi \neq 0$, and we shall expand around the background (2.6) in the so-called "generalized longitudinal gauge" [17], which extends the longitudinal gauge of standard cosmology [36] to the brane-world scenario. As discussed in [17], in five dimensions there are four independent degrees of freedom for the scalar metric fluctuations: in the generalized longitudinal gauge they are described by the four variables $\{\varphi, \psi, \Gamma, W\}$, defined by

$$
\begin{array}{ll}
h_{00}=2 \varphi a^{2}, & h_{i j}=2 \psi a^{2} \delta_{i j}, \\
h_{44}=2 \Gamma a^{2}, & h_{04}=-W a^{2} .
\end{array}
$$

(Off-diagonal metric fluctuations have been taken into account also in a recent study of linearized gravity in a brane-world background [37]: in that case, however, there are no scalar sources in the bulk, and no long-range scalar interactions).

By inserting the explicit form of the background (2.6) and of the metric fluctuations (3.8) into the perturbed equations (3.2) $-(3.5)$, we obtain the full set of constraints and dynamical equations governing the linearized evolution of the five scalar variables $\{\varphi, \psi, \Gamma, W, \chi\}$. Let us give them in components, starting from the Einstein equations, and using eq. (3.5) for the perturbations of the induced metric.

Equation $(0,0)$ gives:

$$
\begin{aligned}
& 2 \nabla^{2} \psi+3 \psi^{\prime \prime}+\nabla^{2} \Gamma+9 \frac{a^{\prime}}{a} \psi^{\prime}-3 \frac{a^{\prime}}{a} \Gamma^{\prime}-\frac{\phi^{\prime}}{2} \chi^{\prime}-6 \frac{a^{\prime \prime}}{a} \Gamma-\frac{\phi^{\prime 2}}{2} \Gamma \\
& -a^{2} e^{\alpha_{1} \phi} \Lambda \alpha_{1} \chi-\frac{1}{2} a e^{2 \alpha_{2} \phi} T_{3}\left(\Gamma+2 \alpha_{2} \chi\right) \delta(z)=0 .
\end{aligned}
$$

Equation $(i, i)$ gives:

$$
\begin{aligned}
& -\nabla^{2} \varphi-\varphi^{\prime \prime}-2 \ddot{\psi}+\nabla^{2} \psi+2 \psi^{\prime \prime}-\ddot{\Gamma}+\nabla^{2} \Gamma \\
& -3 \frac{a^{\prime}}{a} \varphi^{\prime}-\dot{W}^{\prime}-3 \frac{a^{\prime}}{a} \dot{W}+6 \frac{a^{\prime}}{a} \psi^{\prime}-3 \frac{a^{\prime}}{a} \Gamma^{\prime}-\frac{\phi^{\prime}}{2} \chi^{\prime}-6 \frac{a^{\prime \prime}}{a} \Gamma-\frac{\phi^{\prime 2}}{2} \Gamma \\
& -a^{2} e^{\alpha_{1} \phi} \Lambda \alpha_{1} \chi-\frac{1}{2} a e^{2 \alpha_{2} \phi} T_{3}\left(\Gamma+2 \alpha_{2} \chi\right) \delta(z)=0 .
\end{aligned}
$$


Equation $(i, j)$, with $i \neq j$, gives:

$$
\partial_{i} \partial_{j}(\varphi-\psi-\Gamma)=0 .
$$

Equation $(4,4)$ gives:

$$
\begin{aligned}
& -\nabla^{2} \varphi-3 \ddot{\psi}+2 \nabla^{2} \psi-3 \frac{a^{\prime}}{a} \varphi^{\prime}-3 \frac{a^{\prime}}{a} \dot{W}+9 \frac{a^{\prime}}{a} \psi^{\prime}+\frac{\phi^{\prime}}{2} \chi^{\prime} \\
& -12 \frac{a^{\prime 2}}{a^{2}} \Gamma+\frac{\phi^{\prime 2}}{2} \Gamma-a^{2} e^{\alpha_{1} \phi} \Lambda \alpha_{1} \chi=0 .
\end{aligned}
$$

Equation $(i, 0)$ gives:

$$
\partial_{i}\left(\frac{W^{\prime}}{2}+\frac{3}{2} \frac{a^{\prime}}{a} W+2 \dot{\psi}+\dot{\Gamma}\right)=0
$$

Equation $(4,0)$ gives:

$$
\frac{1}{2} \nabla^{2} W-3 \dot{\psi}^{\prime}+3 \frac{a^{\prime}}{a} \dot{\Gamma}+\frac{\phi^{\prime}}{2} \dot{\chi}=0 .
$$

Equation $(4, i)$ gives:

$$
\partial_{i}\left(\frac{\dot{W}}{2}+\varphi^{\prime}-2 \psi^{\prime}+3 \frac{a^{\prime}}{a} \Gamma+\frac{\phi^{\prime}}{2} \chi\right)=0 .
$$

The dilaton equation gives:

$$
\begin{aligned}
& \square_{5} \chi-\phi^{\prime} \varphi^{\prime}-\phi^{\prime} \dot{W}+3 \phi^{\prime} \psi^{\prime}-\phi^{\prime} \Gamma^{\prime}-6 \frac{a^{\prime} \phi^{\prime}}{a} \Gamma-2 \phi^{\prime \prime} \Gamma \\
& +2 a^{2} e^{\alpha_{1} \phi} \Lambda \alpha_{1}^{2} \chi+2 a e^{2 \alpha_{2} \phi} T_{3} \alpha_{2}\left(\Gamma+2 \alpha_{2} \chi\right) \delta(z)=0
\end{aligned}
$$

(the dots denote differentiation with respect to Minkowski time on the brane). Finally, the brane perturbation equation gives a constraint at $z=0$ which is always satisfied because of the $Z_{2}$ symmetry. A similar set of equations was already derived in [17. We have no contributions from the time derivatives of the background, since our background is static.

In the absence of bulk sources with anisotropic stresses, we can now eliminate $\varphi$ from eq. $(i \neq j)$, thus reducing to four scalar degrees of freedom, by setting:

$$
\varphi=\psi+\Gamma
$$

As a consequence, we immediately find that the variable $W$ decouples from the other fluctuations. The combination of the time derivative of eq. $(4, i)$ with the $z$-derivative of eq. $(i, 0)$ and with eq. $(4,0)$ leads in fact to the equation

$$
\square_{5} W=3\left(\frac{a^{\prime \prime}}{a}-\frac{a^{\prime 2}}{a^{2}}\right) W .
$$


Thus $W$ is decoupled but, because of the non-trivial self-interactions, it does not freely propagate in the background geometry like the graviton, eq. (3.7).

In order to discuss the dynamics of the remaining variables $\psi, \Gamma$ and $\chi$, it is now convenient to recombine their differential equations in an explicitly covariant way, to obtain a canonical evolution equation. To this aim, we can use eq. (3.17) and eq. (4,i) to eliminate $\varphi$ and $\dot{W}$ in eqs. $(i, i),(4,4)$ and in the dilaton equation (3.16). We then combine the simplified version of eqs. $(4,4),(i, i)$ with eq. $(0,0)$, and obtain the following system of coupled equations, where the source terms depend only on $\Gamma$ and $\chi$ :

$$
\begin{aligned}
\square_{5} \psi & =-2 \frac{a^{\prime \prime}}{a} \Gamma+2 \frac{a^{\prime 2}}{a^{2}} \Gamma+\frac{a^{\prime} \phi^{\prime}}{a} \chi-\frac{2}{3} a^{2} e^{\alpha_{1} \phi} \Lambda \alpha_{1} \chi \\
& -\frac{1}{6} a e^{2 \alpha_{2} \phi} T_{3}\left(\Gamma+2 \alpha_{2} \chi\right) \delta(z), \\
\square_{5} \Gamma & =-2 \frac{a^{\prime \prime}}{a} \Gamma+8 \frac{a^{\prime 2}}{a^{2}} \Gamma-\phi^{\prime 2} \Gamma+\phi^{\prime \prime} \chi+\frac{a^{\prime} \phi^{\prime}}{a} \chi-\frac{2}{3} a^{2} e^{\alpha_{1} \phi} \Lambda \alpha_{1} \chi \\
& -\frac{2}{3} a e^{2 \alpha_{2} \phi} T_{3}\left(\Gamma+2 \alpha_{2} \chi\right) \delta(z), \\
\square_{5} \chi & =2 \phi^{\prime \prime} \Gamma-\phi^{\prime 2} \chi-2 a^{2} e^{\alpha_{1} \phi} \Lambda \alpha_{1}^{2} \chi \\
& -2 a e^{2 \alpha_{2} \phi} T_{3} \alpha_{2}\left(\Gamma+2 \alpha_{2} \chi\right) \delta(z) .
\end{aligned}
$$

This system can be diagonalized by introducing the fields

$$
\omega_{1}=2 \psi+\Gamma, \quad \omega_{2}=6 \alpha_{2} \Gamma+\chi, \quad \omega_{3}=\Gamma-2 \alpha_{2} \chi,
$$

relations that can be inverted as:

$$
\begin{aligned}
& \psi=\frac{\omega_{1}}{2}-\frac{2 \alpha_{2} \omega_{2}+\omega_{3}}{2\left(1+12 \alpha_{2}^{2}\right)}, \quad \Gamma=\frac{2 \alpha_{2} \omega_{2}+\omega_{3}}{\left(1+12 \alpha_{2}^{2}\right)} \\
& \chi=\frac{\omega_{2}-6 \alpha_{2} \omega_{3}}{\left(1+12 \alpha_{2}^{2}\right)} .
\end{aligned}
$$

In terms of these new variables, the perturbation equations (3.19)-(3.21) reduce to

$$
\begin{aligned}
\square_{5} \omega_{1} & =0, \\
\square_{5} \omega_{2} & =0, \\
\square_{5} \omega_{3} & =\left[-\frac{\phi^{\prime \prime}}{2 \alpha_{2}}-\phi^{\prime 2}-\frac{a^{\prime} \phi^{\prime}}{2 \alpha_{2} a}+\left(\frac{\alpha_{1}}{3 \alpha_{2}}-2 \alpha_{1}^{2}\right) a^{2} e^{\alpha_{1} \phi} \Lambda\right] \omega_{3} \\
& +\left[\left(\frac{2}{3}-4 \alpha_{2}^{2}\right) a e^{2 \alpha_{2} \phi} T \delta(z)\right] \omega_{3} .
\end{aligned}
$$

Together with eq. (3.18), and the constraints (3.13)-(3.15), the above decoupled equations describe the complete evolution of the scalar (metric + dilaton) fluctuations in the CLP brane-world background (2.12), (2.13). Two variables $\left(\omega_{1}, \omega_{2}\right)$ are (covariantly) free 
on the background like the graviton, while the other two variables $\left(\omega_{3}, W\right)$ have non-trivial self-interactions.

In all cases, we can introduce the corresponding "canonical variables" $\widehat{W}, \widehat{\omega}_{i}(i=1,2,3)$, which have canonically normalized kinetic terms [36] in the action, simply by absorbing the geometric warp factor as follows:

$$
W=\widehat{W} a^{-3 / 2}, \quad \omega_{i}=\widehat{\omega}_{i} a^{-3 / 2} .
$$

The variables $\widehat{W}, \widehat{\omega}_{i}$ are required for a correct normalization of the scalar perturbations to a quantum fluctuation spectrum, as they satisfy canonical Poisson (or commutation) brackets. When the general solution is written as a superposition of free, factorized plane-waves modes on the brane,

$$
\widehat{W}=\Psi_{w}(z) e^{-i p_{\mu} x^{\mu}}, \quad \widehat{\omega}_{i}=\Psi_{i}(z) e^{-i p_{\mu} x^{\mu}},
$$

they define the inner product of states with measure $d z$ [18], as in conventional onedimensional quantum mechanics, $\int d z|\Psi(z)|^{2}$.

The allowed mass spectrum of $m^{2}=\eta^{\mu \nu} p_{\mu} p_{\nu}$, for the scalar fluctuations on the brane, can then be obtained by solving an eigenvalue problem in the Hilbert space $L^{2}(R)$ for the canonical variables $\Psi_{w}, \Psi_{i}$, satisfying a Schrödinger-like equation in $z$, which is obtained from the equations (3.18), (3.24)-(3.26) for $W$ and $\omega_{i}$, and which can be written in the conventional form as:

$$
\Psi_{w}^{\prime \prime}+\left(m^{2}-\frac{\xi_{w}^{\prime \prime}}{\xi_{w}}\right) \Psi_{w}=0, \quad \quad \Psi_{i}^{\prime \prime}+\left(m^{2}-\frac{\xi_{i}^{\prime \prime}}{\xi_{i}}\right) \Psi_{i}=0
$$

Here, by analogy with cosmological perturbation theory [36], we have introduced four "pump fields" $\xi_{w}, \xi_{i}$, defined as follows:

$$
\begin{array}{cc}
\xi_{w}=a^{\beta_{w}}, & \xi_{i}=a^{\beta_{i}}, \\
\beta_{w}=-\frac{3}{2}, & \beta_{1}=\beta_{2}=\frac{3}{2},
\end{array}
$$

The effective potential generated by the derivatives of the pump fields depends on $\beta_{w}, \beta_{i}$, and contains in general a smooth part, peaked at $z=0$, plus a positive or negative $\delta$-function contribution at the origin. We may have, in principle, not only volcano-like potentials, which correspond to the free covariant d'Alembert equation with $\beta=3 / 2$ [31] (and which are known to localize gravity [10, 18]), but also potentials that are positive everywhere and admit no bound states. The possible localization of scalar interactions on the 3-brane, for the given background and perturbation equations, will be discussed in the next section. 


\section{Localization of the massless modes}

The general solutions of the canonical perturbation equations $(3.29)$ are labelled by the mass eigenvalue $m$, by their parity with respect to $z$-reflections, and by the parameters $\beta_{w}, \beta_{i}$, which depend on the type of perturbation. The massless scalar modes corresponding to bound states of the effective potential [10, 18, 31] will describe long-range scalar interactions confined to the four-dimensional brane. Concerning $z$-reflections, we shall follow the perturbative formalism developed in [17] consistently, restricting ourselves to a perturbed background that is still $Z_{2}$-symmetric, namely to $Z_{2}$-odd solutions for $W$, and to $Z_{2}$-even solutions for $\omega_{i}$. To describe a bound state, we shall further restrict such solutions to those with a normalizable canonical variable w.r.t. the measure $d z$, namely to $\Psi(z) \in L^{2}(R)$. Among the acceptable solutions, we shall finally select those satisfying the constraints (3.13)-(3.15) derived in the previous section. The above set of conditions will determine the class of brane-world backgrounds allowing the four-dimensional localization of long-range scalar interactions.

For $m=0$ the exact solution of the canonical equations (3.29), for a generic pump field $\xi(z)$, can be separated into an even and an odd part, $\Psi^{+}$and $\Psi^{-}$, as follows:

$$
\Psi_{0}^{+}(z)=c_{+} \xi(z), \quad \Psi_{0}^{-}(z)=c_{-} \xi(z) \int d z^{\prime} \xi^{-2}\left(z^{\prime}\right),
$$

where $c_{+}$and $c_{-}$are integration constants. In order to parametrize the solutions for different values of $\Delta$ and $\beta$ (and also in view of subsequent applications to the massive mode solutions), it is now convenient to introduce the two indices $\nu$ and $\nu_{0}$, defined by

$$
\nu_{0}=\frac{\Delta}{2(\Delta+2)}, \quad \nu=\frac{1}{2}-\frac{2 \beta}{3(\Delta+2)} .
$$

For the three possible values of $\beta_{w}, \beta_{i}$ (see eq. (3.30)), they are related by

$$
\begin{array}{ll}
\nu=1-\nu_{0}, & \beta=\beta_{w}=-\frac{3}{2}, \\
\nu=\nu_{0}, & \beta=\beta_{1,2}=\frac{3}{2}, \\
\nu=2-\nu_{0}, & \beta=\beta_{3}=-\frac{3}{2}(\Delta+3) .
\end{array}
$$

We recall that, in the class of backgrounds we are considering, the parameter $\Delta$ is constrained in the range $-8 / 3 \leq \Delta \leq-2$ (because of the conditions of positive tension, absence of naked singularities and reality of the dilaton couplings). As a consequence, the index $\nu_{0}$ can range from 2 to $+\infty$.

Let us first discuss the limiting case $\nu=\nu_{0}=\infty$, corresponding to $\Delta=-2$. For a generic pump field, with exponent $\beta$, the massless solutions are:

$$
\Psi_{0, \infty}^{+}(z)=c_{0, \infty}^{+} e^{-\frac{\beta}{3} k|z|}, \quad \Psi_{0, \infty}^{-}(z)=c_{0, \infty}^{-} \operatorname{sgn}\{z\}\left(e^{\frac{\beta}{3} k|z|}-e^{-\frac{\beta}{3} k|z|}\right) .
$$


In our background $k>0$, so that the fluctuations $W$ and $\omega_{3}$, both with $\beta=-3 / 2$, are not normalizable. The even solutions of the free d'Alembert equation, with $\beta=3 / 2$, are instead normalizable, so we have acceptable solutions for $\omega_{1}$ and $\omega_{2}$. However, from the constraint $(i, 0)$ of eq. (3.13), $\omega_{1}$ is forced to vanish when $W=0$, unless the fluctuations are static, $\dot{\omega}_{1}=0$. All the other constraints (using also the background equations) are instead identically satisfied by $\omega_{2}$. It follows that, for $\Delta=-2$, there are two independent massless modes localized on the brane: one, $\omega_{2}$, is propagating and the other, $\omega_{1}$, is static.

The same is true for the case $\Delta<-2$, i.e. finite $\nu_{0}$. In that case the massless solutions can be written in the form

$$
\begin{aligned}
& \Psi_{0, \nu}^{+}(z)=c_{0, \nu}^{+}(1+k|z|)^{\frac{1}{2}-\nu}, \\
& \Psi_{0, \nu}^{-}(z)=c_{0, \nu}^{-} \operatorname{sgn}\{z\}\left[(1+k|z|)^{\frac{1}{2}+\nu}-(1+k|z|)^{\frac{1}{2}-\nu}\right] .
\end{aligned}
$$

The solutions for $W$ and $\omega_{3}$ always correspond to $\nu<0$, and are not normalizable. The even d'Alembert modes, with $\nu=\nu_{0}$, are normalizable for $\nu>1$, i.e. $\Delta>-4$, so that $\omega_{1}$ and $\omega_{2}$ are always acceptable in our class of backgrounds, for which $\Delta \geq-8 / 3$. Again, however, the constraint (3.13) implies $\omega_{1}=0$, unless $\dot{\omega}_{1}=0$, while the other constraints are identically satisfied by $\omega_{2}$, so we are left with non-trivial massless solutions only for a propagating fluctuation, $\omega_{2}$, and for a static one, $\omega_{1}$.

We may thus conclude that all backgrounds of the class defined by the conditions (2.14) localize on the brane not only the massless spin-2 degrees of freedom [31] (long-range tensor interactions), but also one propagating massless scalar degree of freedom $\left(\omega_{2}\right)$, corresponding to a long-range scalar interaction generated by the dilaton field. In the longitudinal gauge, the canonical representation $\omega_{2}$ of such a scalar interaction is associated not only with the dilaton fluctuation $\chi$, but also with the four-dimensional "Bardeen potential" $\psi$, and with the "breathing mode" $\Gamma$ of the dimension orthogonal to the brane (see eqs. (3.23)). In addition, we have a second independent massless degree of freedom localized on the brane $\left(\omega_{1}\right)$, which is not propagating $\left(\dot{\omega}_{1}=0\right)$, but is essential to reproduce the standard long-range gravitational interaction in the static limit, as we shall discuss in Section 6.

The localization of the scalar interactions does not impose any further constraints on the background, besides those of eq. (2.14). Also, in the limiting case of a pure $\mathrm{AdS}_{5}$ solution $\left(\Delta=-8 / 3, \nu_{0}=2, \alpha_{1}=0=\alpha_{2}\right)$ the dilaton background disappears, and the dilaton fluctuation $\chi=\omega_{2}$ decouples from the others. The only (static) contribution to the scalar sector of metric fluctuations comes from $\omega_{1}$, which generates the long-range Newton potential $\varphi=\psi$ on the brane (see Section 6). However, even in the case $\Delta=-8 / 3$, the propagating dilaton flucutuation $\omega_{2}$ is non-vanishing, and remains there to describe a (possibly dangerous) long-range scalar interaction. By contrast, no propagating massless 
scalar modes appear in the "pure-gravity" models without bulk scalar fields in the action, as the $\mathrm{AdS}_{5}$ brane-world scenario discussed in 10.

\section{The massive mode spectrum}

The massive part of the spectrum of the canonical equations (3.29) is not localized on the brane; it may induce higher-dimensional (and short-range) corrections to the long-range scalar forces (just as in the case of the pure tensor part of the gravitational interaction [10, 18, 31]). Such corrections thus provide physical effects from the fifth dimension on our brane.

In order to determine the massive modes able to survive the constraints of Section 3, and to evaluate the corresponding short-range corrections, we shall first present the exact solutions of the massive canonical equations (3.29), distinguishing, as before, the even and odd parts under $z$-reflections. For $m \neq 0, \Delta=-2$ (i.e. $\nu_{0}=\infty$ ), and a generic pump field with power $\beta$, such solutions can be written as follows

$$
\begin{aligned}
& \Psi_{m, \infty}^{+}(z)=\frac{1}{\sqrt{\pi m q}}\left(q \cos q|z|-\frac{\beta}{3} k \sin q|z|\right), \\
& \Psi_{m, \infty}^{-}(z)=-\sqrt{\frac{m}{\pi q}} \sin q z,
\end{aligned}
$$

where

$$
q=\left(m^{2}-\frac{k^{2}}{4}\right)^{1 / 2}
$$

For $\Delta<-2$, i.e. finite $\nu_{0}$, the solution can be written as a combination of first- and second-kind Bessel functions $J_{\nu}$ and $Y_{\nu}$ [38], of index $\nu$ given by eq. (4.2]:

$$
\begin{aligned}
& \Psi_{m, \nu}^{+}(z)=c_{m, \nu-1} \sqrt{1+k|z|}\left[Y_{\nu-1}\left(\frac{m}{k}\right) J_{\nu}(y)-J_{\nu-1}\left(\frac{m}{k}\right) Y_{\nu}(y)\right], \\
& \Psi_{m, \nu}^{-}(z)=c_{m, \nu} \operatorname{sgn}\{z\} \sqrt{1+k|z|}\left[Y_{\nu}\left(\frac{m}{k}\right) J_{\nu}(y)-J_{\nu}\left(\frac{m}{k}\right) Y_{\nu}(y)\right],
\end{aligned}
$$

where

$$
c_{m, \nu}=\sqrt{\frac{m}{2 k}}\left[J_{\nu}^{2}\left(\frac{m}{k}\right)+Y_{\nu}^{2}\left(\frac{m}{k}\right)\right]^{-\frac{1}{2}}, \quad y=\frac{m}{k}(1+k|z|) .
$$

Note that in the above equations we have adopted the $\delta$-function normalization of the continuum modes, as for plane waves in one-dimensional quantum mechanics. As a consequence, $\Psi_{m}$ is dimensionless (unlike in [31], where a different normalization is adopted).

It is important to note that modes with negative squared mass (tachyons) are not included in the spectrum, as they would not correspond to a normalizable canonical variable 
( $\Psi$ would blow up in $z$ ). This can be regarded as a direct check of the stability of the given class of CLP backgrounds against scalar perturbations, since tachyonic modes would also blow up in time, and would destroy the assumed homogeneity of the four-dimensional brane. Another consequence of the normalization condition is the mass gap $\left(m^{2}>k^{2} / 4\right.$, see eq. (5.2)) between the localized massless mode and the massive corrections, in the limiting background with $\nu_{0}=\infty$ (already noticed in [31] for the case of pure tensor interactions).

We shall now impose the constraints (3.13)-(3.15) following from the perturbed background equations. It is convenient to introduce the four amplitudes $A_{i}, A_{w}$, defined by

$$
\begin{array}{rrr}
\omega_{1}=\frac{a^{-3 / 2} A_{1}}{c_{m, \nu_{0}-1}} \Psi_{m, \nu_{0}}^{+}(z) e^{-i p_{\mu} x^{\mu}}, & \omega_{2}=\frac{a^{-3 / 2} A_{2}}{c_{m, \nu_{0}-1}} \Psi_{m, \nu_{0}}^{+}(z) e^{-i p_{\mu} x^{\mu}} \\
\omega_{3}=\frac{a^{-3 / 2} A_{3}}{c_{m, 1-\nu_{0}}} \Psi_{m, 2-\nu_{0}}^{+}(z) e^{-i p_{\mu} x^{\mu}}, & W=\frac{a^{-3 / 2} A_{w}}{c_{m, 1-\nu_{0}}} \Psi_{m, 1-\nu_{0}}^{-}(z) e^{-i p_{\mu} x^{\mu}}
\end{array}
$$

where $\Psi^{ \pm}$are the above normalized solutions. Plugging this ansatz into the constraint equations (3.13)-(3.15), we find that, in contrast with the massless case, none of the four scalar fluctuations is forced to vanish. However, only two amplitudes are independent. By taking, for instance, $\omega_{2}$ and $\omega_{3}$ as independent variables, we can indeed express $A_{1}$ and $A_{w}$ in terms of $A_{2}$ and $A_{3}$, for all values of $\nu_{0}$, in such a way that all the constraints are identically satisfied. For a generic mode of mass $m$ and momentum $p$, we find, in particular,

$$
\begin{aligned}
& A_{1}=\frac{m^{2}}{9 m^{2}+6 p^{2}} \frac{\sqrt{2 \nu_{0}-1}}{\nu_{0}-1}\left(\sqrt{3\left(\nu_{0}-2\right)} A_{2}+3 \sqrt{2 \nu_{0}-1} A_{3}\right) \\
& A_{w}=\frac{2 i m \sqrt{m^{2}+p^{2}}}{9 m^{2}+6 p^{2}} \frac{\sqrt{2 \nu_{0}-1}}{\nu_{0}-1}\left(\sqrt{3\left(\nu_{0}-2\right)} A_{2}+3 \sqrt{2 \nu_{0}-1} A_{3}\right),
\end{aligned}
$$

which also hold when $\nu_{0} \rightarrow \infty$. Note that a single combination of $A_{2}$ and $A_{3}$ determines both $A_{1}$ and $A_{w}$.

For such backgrounds we thus have four types of higher-dimensional contributions to the scalar interactions on the brane, arising from the massive spectrum of $\omega_{i}$ and $W$. The exchange of such massive modes generates corrections to the four-dimensional scalar forces. The corrections are in general different from those of tensor interactions, arising from the massive spectrum of a variable satisfying the free d'Alembert equation, like $\omega_{2}$. In the weak field limit, however, the leading-order contributions to the non-relativistic potential generated by a static scalar sources have the same qualitative behaviour as in the case of tensor interactions, as will be illustrated in the next section. 


\section{$6 \quad$ Static limit and leading-order corrections}

To make contact with previous works, and for future phenomenological applications, we shall now compute the effective scalar-tensor interaction induced on the brane, in the weak field limit, by a static and point-like source of mass $M$ and dilatonic charge $Q$.

In our longitudinal gauge (3.6), (3.8), in which the decomposition of the metric fluctuations is based on the $O(3)$ symmetry of the spatial hypersurfaces of the brane, the energy density of a point-like particle only contributes to the scalar part of the perturbed matter stress tensor (with $T_{00}$ as the only non-vanishing component), and provides a $\delta$-function source to the $(0,0)$ scalar perturbation equation (3.9). Similarly, the charge $Q$ acts as a point-like source in the dilaton perturbation equation (3.16).

As a consequence, we obtain three $\delta$-function sources in the equations for the three $\omega_{i}$ fluctuations, $S_{i} \delta^{3}\left(x-x^{\prime}\right) \delta(z)$, with three scalar charges $S_{i}$, which are "mixtures" of $M$ and $Q$, while no source term is obtained in the static limit for the $W$ fluctuation (in agreement with the fact that $W=0$, in the static limit, according to the constraint $(i, 0))$. The effective sources $S_{i}$ for the massless and massive $\omega_{i}$ fluctuations are defined by the combination of eqs. $(4,4),(i, i),(0,0)$, and by the dilaton equation (3.16), as follows:

$$
S_{1}=M, \quad S_{2}=Q+2 \alpha_{2} M, \quad S_{3}=\frac{M}{3}-2 \alpha_{2} Q
$$

The exact static solutions of eqs. (3.24)-(3.26), with the above point-like sources, can be easily obtained using the static limit of the retarded Green function evaluated on the brane $(z=0)$, i.e.

$$
\omega_{i}\left(\nu, x, x^{\prime}\right)=-S_{i} G_{i}\left(\nu, x, x^{\prime}\right),
$$

where

$$
G_{i}\left(\nu, \vec{x}, \vec{x}^{\prime}, z=z^{\prime}=0\right)=\int \frac{d^{3} p}{(2 \pi)^{3}} e^{i \vec{p} \cdot\left(\vec{x}-\vec{x}^{\prime}\right)}\left\{\frac{\left[\psi_{0, \nu}^{+}(0)\right]^{2}}{p^{2}}+\int_{m_{0}}^{\infty} d m \frac{\left[\psi_{m, \nu}^{+}(0)\right]^{2}}{p^{2}+m^{2}}\right\}
$$

(see also [31, 32, 39]). Here $\nu=\nu_{0}$ for $i=1,2$, while $\nu=2-\nu_{0}$ for $i=3$, and $\psi_{0, \nu}^{+}(z), \psi_{m, \nu}^{+}(z)$ (including the case $\nu=\infty$ ) are the exact solutions (4.4), (4.5), (5.1), (5.3), obtained in the previous sections. The first term in the integrand corresponds to the long-range forces generated by the massless modes, the second term to the "short-range" corrections due to the massive modes, and $m_{0}$ is the lower bound for the massive spectrum $\left(m_{0}=k / 2\right.$ if $\nu_{0}=\infty$, while $m_{o}=0$ if $\nu_{0}<\infty$, see eqs. (5.1), (5.2)).

We should note that in the $\omega_{1}, \omega_{2}$ case we have to include both the massless and massive contributions, while in the $\omega_{3}$ case only the massive ones survive (indeed, we recall that 
for $m=0$ the $\omega_{3}$ modes are not normalizable, and that the massless sector of $\omega_{1}$, in the static limit, is not eliminated by the constraint $(i, 0)$, see Section 4$)$. We also note that the amplitude of the massless solutions (4.4), (4.5) has to be fixed by the correct normalization, i.e.

$$
c_{0, \infty}^{+}=(k \beta / 3)^{1 / 2}, \quad c_{0, \nu}^{+}=[k(\nu-1)]^{1 / 2} .
$$

Let us start with $\omega_{1}$, for which $\nu=\nu_{0}$, and with the limiting background $\Delta=-2$, i.e. $\nu=\infty$. By setting $\beta=3 / 2$, and using eqs. (4.4) and (5.1) for $\psi_{0, \infty}^{+}$and $\psi_{m, \infty}^{+}$, respectively, we obtain

$$
\omega_{1}(\nu=\infty)=-S_{1} \frac{k}{8 \pi r}\left[1+\frac{2}{k \pi} \int_{k / 2}^{\infty} \frac{d m}{m}\left(m^{2}-\frac{k^{2}}{4}\right)^{1 / 2} e^{-m r}\right],
$$

where $r=\left|\vec{x}-\vec{x}^{\prime}\right|$. The same integral had already been obtained in [31] when discussing the localization of tensor fluctuations, and the associated leading-order corrections (in the large-distance limit) are known to be of the form $e^{-k r / 2}(k r)^{-3 / 2}$.

In the case $\Delta<-2$, i.e. $\nu=\nu_{o}<\infty$, we shall use instead eqs. (4.5) and (5.3) for $\psi_{0, \nu_{0}}^{+}$and $\psi_{m, \nu_{0}}^{+}$, respectively. Also, we shall estimate the contribution of the massive modes by expanding the Bessel functions in the small- $m$ regime (which is relevant at the long distances typical of the weak field limit). The small argument limit [38] of $J_{\nu}, Y_{\nu}$ then gives

$$
\left[\psi_{m, \nu_{0}}^{+}(0)\right]^{2}=\frac{1}{\Gamma^{2}\left(\nu_{0}-1\right)}\left(\frac{m}{2 k}\right)^{2 \nu_{0}-3}
$$

and we are led to

$$
\omega_{1}(\nu<\infty)=-S_{1} \frac{k\left(\nu_{0}-1\right)}{4 \pi r}\left[1+\frac{\Gamma\left(\nu_{0}-1 / 2\right)}{\sqrt{\pi} \Gamma\left(\nu_{0}\right)}\left(\frac{1}{k r}\right)^{2 \nu_{0}-2}\right]
$$

(here $\Gamma$ obviously represents the Euler function, not to be confused with the $g_{44}$ component of the metric fluctuations).

Exactly the same results (6.5), (6.7) are obtained for $\omega_{2}$, which satisfies the same free d'Alembert equation as $\omega_{1}$, with the only difference that $S_{1}$ has to be replaced by $S_{2}$.

Let us then compute $\omega_{3}$, for which $\nu=2-\nu_{0}$, and which has only the massive mode contribution to the Green function. For $\Delta=-2$, i.e $\nu=\infty$, we shall use eq. (5.1) for $\psi_{m, \infty}^{+}$. At $z=0$ the solution however is $\beta$-independent, and we obtain the (massive part) of the result already given in eq. (6.5) (with $S_{1}$ replaced by $S_{3}$ ).

In the case $\Delta<-2$, i.e $\nu<\infty$, we shall use $\psi_{m, 2-\nu_{0}}^{+}$from eq. (5.3). In the large-distance (small- $m$ ) regime, however, the contribution at $z=0$ to the Green function is exactly the same as that of eq. (6.6), so that we obtain

$$
\omega_{3}(\nu<\infty)=-S_{3} \frac{k\left(\nu_{0}-1\right)}{4 \pi r} \frac{\Gamma\left(\nu_{0}-1 / 2\right)}{\sqrt{\pi} \Gamma\left(\nu_{0}\right)}\left(\frac{1}{k r}\right)^{2 \nu_{0}-2} .
$$


We are now ready to estimate, in the static limit, the dilaton and metric fluctuations $\chi, \Gamma, \psi, \varphi$. We shall explicitly consider the case $\nu_{0}<\infty$, for an easy comparison with previous results relative to an $\mathrm{AdS}_{5}$ background, for which $\nu_{0}=2$. Defining

$$
A_{\nu_{0}}=\frac{k\left(\nu_{0}-1\right)}{4 \pi}, \quad B_{\nu_{0}}=\frac{\Gamma\left(\nu_{0}-1 / 2\right)}{\sqrt{\pi} \Gamma\left(\nu_{0}\right)},
$$

we first rewrite the $\omega_{i}$ solutions in compact form as:

$$
\begin{aligned}
& \omega_{1}=-\frac{S_{1} A_{\nu_{0}}}{r}\left[1+B_{\nu_{0}}\left(\frac{1}{k r}\right)^{2 \nu_{0}-2}\right], \\
& \omega_{2}=-\frac{S_{2} A_{\nu_{0}}}{r}\left[1+B_{\nu_{0}}\left(\frac{1}{k r}\right)^{2 \nu_{0}-2}\right], \\
& \omega_{3}=-\frac{S_{3} A_{\nu_{0}}}{r} B_{\nu_{0}}\left(\frac{1}{k r}\right)^{2 \nu_{0}-2} .
\end{aligned}
$$

We may note, as a check of our previous computations, that the relative amplitude of the massive corrections, in the weak, static limit, is controlled by the correspondig scalar charges, which satisfy the relation (from eq. 6.1)):

$$
S_{3}+2 \alpha_{2} S_{2}=\left(\frac{1}{3}+4 \alpha_{2}^{2}\right) S_{1}
$$

By eliminating $\alpha_{2}$ in favour of $\Delta$ according to eq. (2.11), and $\Delta$ in terms of $\nu_{0}$, according to eq. (4.2), we obtain

$$
S_{1}=\frac{1}{\nu_{0}-1}\left[\frac{1}{3} \sqrt{3\left(\nu_{0}-2\right)\left(2 \nu_{0}-1\right)} S_{2}+\left(2 \nu_{0}-1\right) S_{3}\right]
$$

which exactly reproduces, in the static limit $m^{2}+p^{2} \rightarrow 0$, the general relation (5.6) between the massive amplitudes.

It is convenient, at this point, to explicitly introduce the four-dimensional gravitational constant $M_{p}^{-2}$, by noting that

$$
A_{\nu_{0}}=\frac{k\left(\nu_{0}-1\right)}{4 \pi}=\frac{1}{4 \pi}\left[\psi_{0, \nu_{0}}^{+}(0)\right]^{2}=\frac{1}{4 \pi}\left[\int d z a^{3}(z)\right]^{-1} .
$$

The above normalization integral, when expressed in terms of a new bulk coordinate $y$, with $d y=a(z) d z$, represents the warped extra-dimensional volume that controls the ratio between the four- and five-dimensional gravitational constants [9, 10], i.e.

$$
M_{p}^{2}=M_{5}^{3} \int d y a^{2}(y)=M_{5}^{3} \int d z a^{3}(z)
$$

It follows that, in units such that $M_{5}^{3}=1$,

$$
A_{\nu_{0}}=\left(4 \pi M_{p}^{2}\right)^{-1}=2 G
$$


where $G$ is the Newton coupling constant. The scalar and dilaton fluctuations (3.23), using the static solutions 6.10, can then be written in the form:

$$
\begin{aligned}
& \varphi=-\frac{G M}{r}\left[1+\frac{2 \alpha_{2}}{1+12 \alpha_{2}^{2}}\left(\frac{Q}{M}+2 \alpha_{2}\right)+\frac{4}{3} B_{\nu_{0}}\left(\frac{1}{k r}\right)^{2 \nu_{0}-2}\right], \\
& \psi=-\frac{G M}{r}\left[1-\frac{2 \alpha_{2}}{1+12 \alpha_{2}^{2}}\left(\frac{Q}{M}+2 \alpha_{2}\right)+\frac{2}{3} B_{\nu_{0}}\left(\frac{1}{k r}\right)^{2 \nu_{0}-2}\right], \\
& \Gamma=-\frac{G M}{r}\left[\frac{4 \alpha_{2}}{1+12 \alpha_{2}^{2}}\left(\frac{Q}{M}+2 \alpha_{2}\right)+\frac{2}{3} B_{\nu_{0}}\left(\frac{1}{k r}\right)^{2 \nu_{0}-2}\right], \\
& \chi=-\frac{G Q}{r}\left[\frac{2}{1+12 \alpha_{2}^{2}}\left(1+2 \alpha_{2} \frac{M}{Q}\right)+2 B_{\nu_{0}}\left(\frac{1}{k r}\right)^{2 \nu_{0}-2}\right] .
\end{aligned}
$$

It should be noted that the short-range corrections induced by the massive scalar modes have the same qualitative behaviour as in the tensor case, discussed in 31, in spite of the fact that the massive scalar modes have different spectra. The dimensional decoupling (i.e., the suppression of the higher-dimensional corrections) is thus effective for all scales of distance $r$ such that $k r \gg 1$, where $k=\left(M_{5}^{3} / M_{p}^{2}\right)\left(\nu_{0}-1\right)^{-1}$ is the mass scale relating the five- and four-dimensional gravitational constants, in our class of backgrounds.

The limiting case $\Delta=-8 / 3$, i.e. $\alpha_{2}=0$ and $\nu_{0}=2$, corresponds to a pure $\mathrm{AdS}_{5}$ background, if there are no scalar charges on the brane. In that case $\omega_{2}$ exactly corresponds to the dilaton fluctuation $\chi$ (see eq. (3.22)), and can be consistently set to zero (toghether with the dilaton background) if we want to match, in particular, the "standard" brane-world configuration originally considered by Randall and Sundrum [10]. In this limit, $B_{2}=1 / 2$, and we exactly recover previous results for the effective gravitational interaction on the brane [32], i.e.

$$
\varphi=-\frac{G M}{r}\left(1+\frac{2}{3 k^{2} r^{2}}\right), \quad \psi=-\frac{G M}{r}\left(1+\frac{1}{3 k^{2} r^{2}}\right)
$$

The massless-mode truncation reproduces in this case the static, weak field limit of linearized general relativity (note, however, that for $Q \neq 0$ there is no way to get rid of the long-range scalar interactions). The massive tower of scalar fluctuations, however, induces deviations from Einstein gravity already in the static limit (as noted in [32]), and is the source of a short-range force due to the "breathing" of the fifth dimension,

$$
\Gamma=-\frac{G M}{3 r} \frac{1}{(k r)^{2}},
$$

even in the absence of bulk scalar fields, and of scalar charges for the matter on the brane.

In a more general gravi-dilaton background $\left(\Delta \neq-8 / 3, \alpha_{2} \neq 0\right)$, the static expansion (6.16) describes an effective scalar-tensor interaction on the brane, which is potentially 
dangerous for the brane-world scenario, as it contains not only short-range corrections, but also long-range scalar deviations from general relativity (and, possibly, violations of the Einstein equivalence principle), even in the interaction of ordinary masses, i.e. for $Q=0$ (similar results have been recently obtained also in the context of a multibrane scenario [40]). This seems to offer an interesting window to investigate the effects of the bulk geometry on the four-dimensional physics of the brane.

\section{Conclusions}

In this paper we have analysed the full set of coupled equations governing the evolution of scalar fluctuations in a dilatonic brane-world background, supporting a flat 3-brane rigidly located at the fixed point of $Z_{2}$ symmetry. We have diagonalized the system of dynamical equations, and found four decoupled but self-interacting variables representing, in a fivedimensional bulk, the four independent degrees of freedom of scalar excitations of the gravidilaton background.

We have then restricted our discussion to the class of background solutions characterized by a brane of positive tension, by a decreasing warp-factor as we move away from the brane, and by the absence of naked singularities [31. Such a class of backgrounds can be characterized by a real parameter $\Delta$ ranging from $-8 / 3$ to -2 or, alternatively, by a real parameter $\nu_{0}$ ranging from 2 to $+\infty$. The limiting case $\Delta=-8 / 3, \nu_{0}=2$, corresponds to the "pure-gravity" $\mathrm{AdS}_{5}$ background [10].

We have presented the exact solutions of the canonical perturbation equation for all the scalar degrees of freedom, and we have discussed, in this class of backgrounds, the effects of their massless and massive spectrum for the scalar interactions on the brane, taking into account the appropriate parity under $Z_{2}$ symmetry, the normalization condition for the bound states of the spectrum, and the first-order differential constraints arising from the dynamics of scalar perturbations.

We have found that, for all backgrounds, there is one propagating massless mode localized on the brane, associated with a long-range dilatonic interaction in four dimensions. Only very exceptionally (i.e. for a RS background and vanishing dilatonic charges) this "fifth force" disappears. This interaction is always affected by higher-dimensional corrections, due to the scalar massive modes that are not confined on the brane and can freely propagate in the bulk space-time. The amplitudes of such massive modes are constrained by the scalar perturbation equations and, in general, only two amplitudes can be independently assigned. The scalar fluctuation spectra are in general different from the corresponding spectra of the spin-2 degrees of freedom. In the weak and static limit, however, the leading-order short- 
range corrections to the scalar force have the same radial dependence as in the case of pure tensor interactions.

In particular, we have found a non-trivial massive spectrum of scalar metric fluctuations even in the pure Randall-Sundrum scenario with $\mathrm{AdS}_{5}$ metric [10]. In more general backgrounds we have found that there are also scalar contributions to the long-range interactions of two massive bodies, even in the absence of specific "dilatonic" charges, with a resulting effective scalar-tensor interaction on the brane. In this sense, the bulk geometry seems to affect not only the radial dependence, but also the spin content of the effective gravitational

forces. We believe that this effect is potentially interesting for further applications of (and constraints on) the brane-world scenario.

\section{Acknowledgements}

It is a plesure to thank P. Binétruy, M. Giovannini, D. Langlois and C. Ungarelli for helpful conversations. G.V. wishes to acknowledge the support of a "Chaire Internationale Blaise Pascal", administered by the "Fondation de L'Ecole Normale Supérieure", during most of this work. 


\section{References}

[1] P. Horava and E. Witten, Nucl. Phys. B 460, 506 (1996).

[2] P. Horava and E. Witten, Nucl. Phys. B 475, 96 (1996).

[3] A. Lukas, B. A. Ovrut, K. S. Stelle and D. Waldram, Phys. Rev. D59, 086001 (1999).

[4] A. Lukas, B. A. Ovrut, K. S. Stelle and D. Waldram, Nucl. Phys. B552, 246 (1999).

[5] I. Antoniadis, Phys. Lett. B 246, 377 (1990).

[6] N. Arkani-Hamed, S. Dimopoulos and G. Dvali, Phys. Lett. B 429, 263 (1998).

[7] I. Antoniadis, N. Arkani-Hamed, S. Dimopoulos and G. Dvali, Phys. Lett. B 436, 257 (1998).

[8] V. Rubakov and M. E. Shaposhnikov, Phys. Lett. 125B, 139 (1983).

[9] L. Randall and R. Sundrum, Phys. Rev. Lett. 83, 3370 and 4690 (1999).

[10] L. Randall and R. Sundrum, Phys. Rev. Lett. 83, 4690 (1999).

[11] R. B. Abbott, B. Bednarz and S. D. Ellis, Phys. Rev. D 33, 2147 (1986).

[12] J. Garriga and E. Verdaguer, Phys. Rev. D 39, 1072 (1989).

[13] L. Amendola, M. Litterio and F. Occhionero, Phys. Lett. B 237, 348 (1990).

[14] M. Gasperini and M. Giovannini, Phys. Rev. D 47, 1519 (1993).

[15] M. Gasperini and M. Giovannini, Class. Quantum Grav. 14, 735 (1997).

[16] M. Giovannini, Phys. Rev. D 55, 595 (1997).

[17] C. van de Bruck, M. Dorca, R. H. Brandenberger and A. Lukas, Phys. Rev. D 62, 123515 (2000).

[18] C. Csaki, J. Erlich, T. J. Hollowood and T. Shirman, Nucl. Phys. B 581, 309 (2000).

[19] T. R. Taylor and G. Veneziano, Phys Lett. B 213, 459 (1988).

[20] E. Fischbach and C. Talmadge, Nature 356, 207 (1992).

[21] T. Damour and A. M. Polyakov, Nucl. Phys. B 423, 532 (1994).

[22] D. Langlois, Phys. Rev. D 62, 126012 (2000). 
[23] D. Langlois, R. Maartens, M. Sasaki and D. Wands, Phys. Rev. D 63, 084009 (2001).

[24] B. Grinstein, D. R. Nolte and W. Skiba, hep-th/0005001.

[25] N. Deruelle, T. Dolezel and J. Katz, hep-th/0010215.

[26] U. Gen and M. Sasaki, gr-qc/0011078.

[27] A. Neronov and I. Sachs, hep-th/0011254.

[28] C. van de Bruck and M. Dorca, hep-th/0012073.

[29] R. Maartens, gr-qc/0101059.

[30] T. Boehm, R. Durrer and C. van de Bruck, hep-th/0102144.

[31] M. Cvetic, H. Lu and C.N. Pope, Phys. Rev. D 63, 086004 (2001).

[32] J. Garriga and T. Tanaka, Phys. Rev. Lett. 84, 2778 (2000).

[33] M. J. Duff, in Second Conference on Gauge Theories, Applied Supersymmetry and Quantum Gravity (Imperial Coll. Press, London, 1996).

[34] M. J. Duff, Class. Quantum Grav. 5, 189 (1988).

[35] M. Cvetic, S. Griffies and H. H. Soleng, Phys. Rev. D 48, 2613 (1993).

[36] V. F. Mukhanov, H. A. Feldman and R. Brandenberger, Phys. Rep. 215, 203 (1992).

[37] I. Y. Aref'eva et al, Nucl. Phys. B 590, 273 (2000).

[38] M. Abramovicz and I. A. Stegun, Handbook of Mathematical Functions (Dover, New York, 1972).

[39] A. Brandhuber and K. Sfetsos, JHEP 9910, 013 (1999).

[40] I. I. Kogan, S. Mouslopoulos, A. Papazoglou and L. Pilo, hep-th/0105255. 